\title{
Surgical treatment of acute pulmonary embolism: a modified surgical technique to avoid deep hypothermia and circulatory arrest
}

\author{
Emanuele Pilato, Giuseppe Comentale \\ Department of Advanced Biomedical Sciences, Cardiac Surgery, University of Napoli "Federico II", Napoli, Italy \\ Correspondence to: Giuseppe Comentale, MD. Cardiac Surgery, University of Napoli "Federico II”, Via Sergio Pansini n5, 80131, Napoli, Italy. \\ Email: giuseppe.comentale@unina.it.
}

\begin{abstract}
Massive acute pulmonary embolism (MAPE) is a rare and life-threatening condition that cardiothoracic surgeons may face during their clinical practice. As considerable improvements have been made in pharmacological and interventional treatment, MAPE is usually treated nonsurgically. A surgical approach, even if it is proven to be a good and efficacious alternative to thrombolysis, is considered only after a failure of the medical therapy or in the presence of massive occlusion of the main branches of the pulmonary arteries. In order to achieve a good and bloodless view of the operative field, some cardiothoracic surgeons prefer to treat this condition adopting the same technique used for chronic pulmonary embolism recurring to deep hypothermia and circulatory arrest; however, this approach usually leads to neurologic or splanchnic hypoperfusion problems. We here describe a modified surgical technique that does not require deep hypothermia or circulatory arrest, the consequences of which are reductions in surgery time and the postoperative recovery period in the intensive care unit (ICU). Shorter cardiopulmonary bypass times allow for lower hemodilution levels and a decreased inflammatory response, which in turn leads to a decreased risk of cerebral, lung and renal edema and quicker discharge from the ICU.
\end{abstract}

Keywords: Massive pulmonary embolism; pulmonary endarterectomy; deep hypothermia

Received: 22 April 2020; Accepted: 02 July 2020; Published: 25 November 2020.

doi: $10.21037 /$ ccts-20-84

View this article at: http://dx.doi.org/10.21037/ccts-20-84

\section{Introduction}

Massive acute pulmonary embolism (MAPE) is a rare and life-threatening condition that cardiothoracic surgeons may face during their clinical practice. This condition is usually caused by a sudden and extensive occlusion of the main pulmonary artery or more than $60 \%$ of its branches due to a dislodged thrombus that is frequently embolized from the legs or arms during a deep vein thrombosis or after a major trauma. MAPE occurs in $4.2 \%$ of the pulmonary embolism cases (1). Mortality depends on the percentage of pulmonary vascular bed involved and hemodynamic and clinical state, and ranges between $1 \%$ and $36 \%(2,3)$. It can reach $70 \%$ in the presence of hemodynamic instability, when more than the half of pulmonary branches are occluded (4) or if emergency cardiopulmonary bypass $(\mathrm{CPB})$ is required
$(3,5,6)$. As result of the wide pulmonary occlusion, respiratory and cardiac function quickly deteriorate due to increased right ventricle (RV) pressure and reduced left ventricle preload, while marked hypoxia usually occurs in the first hours after the thromboembolic event and requires endotracheal intubation. This is related to the increased alveolar death space caused by blood flow redistribution together with RV depression and ischemia. Vasopressors and phosphodiesterase inhibitors are often enough to sustain the cardiac output, but sometimes peripheral CPB is necessary to stabilize the patients. In these situations, surgical treatment still remains the gold standard technique, and its timing is dictated by the hemodynamic status of the patient $(4,7)$.

The first case of successful surgical treatment for MAPE 

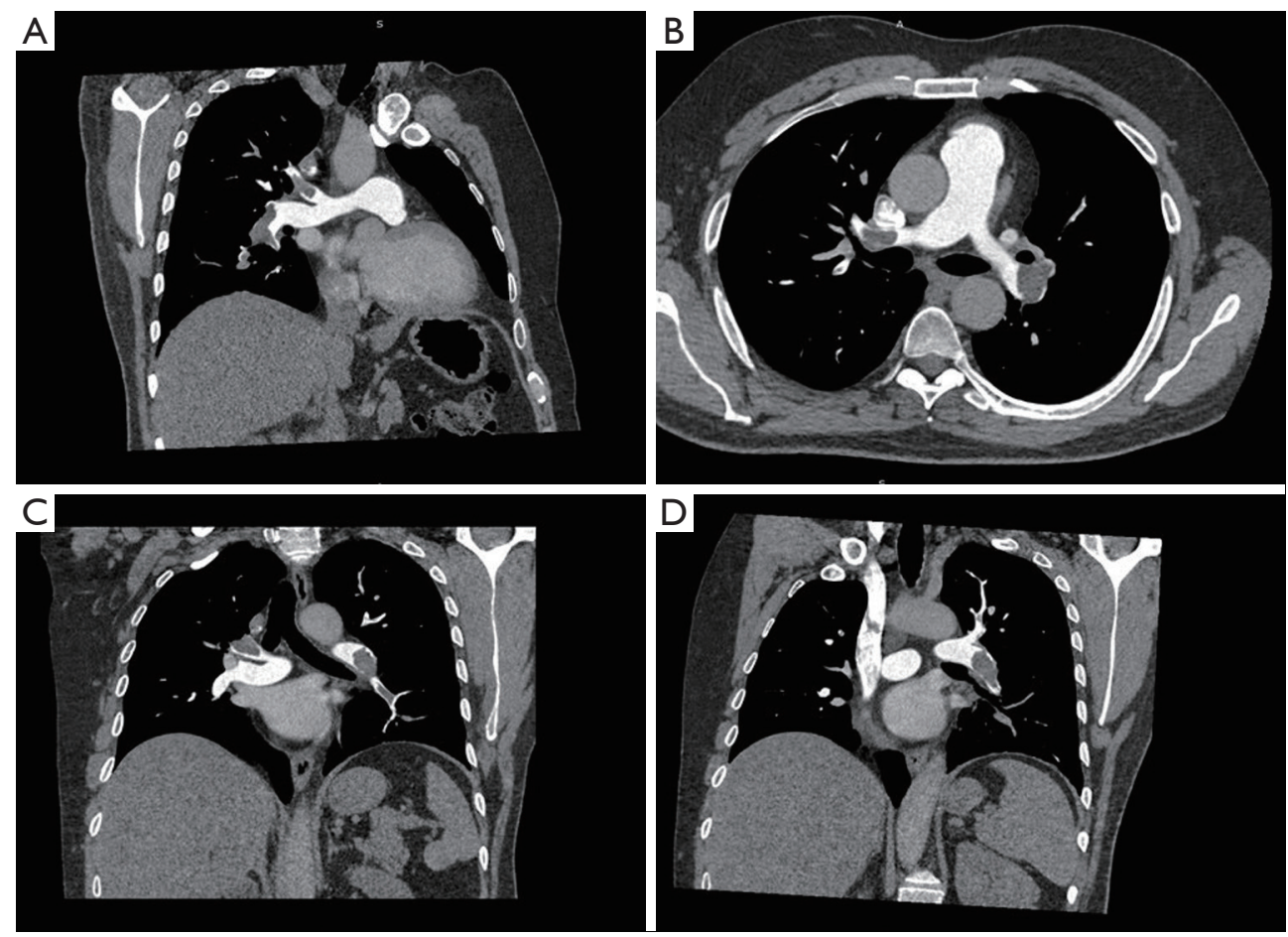

Figure 1 Angio-CT scan showing the subtotal occlusion of the principal pulmonary artery extended to the all lobar arteries and their segmental and subsegmental branches in the two lungs. CT, computed tomography.

was described by Cooley and colleagues in 1961 (8). Since then, the surgical technique has evolved thanks to improvements in materials and to a deeper knowledge of extracorporeal circulation physiology which makes avoiding deep hypothermia and circulatory arrest possible. In order to describe our surgical technique and perioperative management of patients with MAPE, we present the case of a 43-year-old Italian man who was transferred to the cardiac surgery intensive care unit (CICU) due to sudden heart and respiratory failure.

\section{Surgical technique}

\section{Patient's clinical bistory}

The patient was previously hospitalized in the cardiologic intensive care unit (ICU) to treat a diffuse pulmonary embolism that was clinically and radiologically confirmed. A few days prior, he had visited the emergency department due to fever and mild dyspnea, where, according to the chest X-ray pattern, he was treated for infective pneumonia. Clinical conditions quickly deteriorated, and thus a computed tomography angiography (CTA) scan was performed, revealing a wide and bilateral pulmonary embolism. The patient was therefore transferred to the cardiologic ICU to begin appropriate treatment. He was hemodynamically stable, but he presented with dyspnea and cyanosis for mild efforts and had no previous history of actual or recent trauma/deep vein thrombosis. Laboratory and clinical evidence for thrombophilic conditions was also absent. Therefore, continuous unfractionated heparin infusion was started at 1,250 units/h with a targeted activate partial thromboplastin time (aPTT) of at least 80 seconds $(9,10)$. The patient seemed to be resistant to medical treatment, probably as a result of a then unrecognized congenital thrombophilia, and 2 days after he developed marked hypotension, dyspnea, and distension of the neck veins. Echocardiogram revealed a great increase in $\mathrm{RV}$ pressure, a prominent "a" wave and a depressed RV function. An urgent chest CTA scan was performed which showed that both lungs had subtotal occlusion of the principal pulmonary artery extending to all the lobar arteries and their segmental and subsegmental branches (Figure 1). This was in contrast with literature which reports most involvement being in the lower lobes, and more often in the right than the left lung (5). According to recent 
guidelines (9), thrombolytic treatment was initiated but was also ineffectual.

\section{Indications and preoperative management}

The patient was moved to the CICU because of progressive heart and respiratory failure. Endotracheal intubation was performed, and continuous vasopressors infusion was started in order to stabilize hemodynamics and to maintain arterial oxygen pressure $\left(\mathrm{aPO}_{2}\right)$ at a minimum of over $60 \mathrm{mmHg}$. According to the literature (10) and our institution protocol, once the patient was stabilized, urgent surgery was planned for the next day. Meanwhile, continuous unfractionated heparin infusion was started in order to obtain an activated clotting time (ACT) of over 200 seconds. Blood gases analysis was performed each hour, with particular attention being paid to serum lactate levels and $\mathrm{aPO}_{2}$. Increase in serum lactate or decrease in $\mathrm{PO}_{2}$ is considered an indication for imminent surgery. For these reasons, truly massive "acute" pulmonary embolisms that are treated surgically are today very rare because surgery is considered only after medical therapy fails. This approach does indeed confront the surgeon with a "subacute" pattern that, due to the progressive thrombus stratification, requires a surgical technique much more similar to that used in a chronic pulmonary embolism than in an acute one.

\section{Intraoperative strategies}

One of the key points of the MAPE surgery is the management of the $\mathrm{CPB}$, especially with respect to hypothermia and circulatory arrest. Some cardiothoracic surgeons treat this condition using the same technique as that used for chronic pulmonary embolism recurring to deep hypothermia and circulatory arrest (11) but this approach usually leads to neurologic or splanchnic hypoperfusion problems. When this technique is not strictly necessary, in order to avoid these possible complications and to accelerate postoperative recovery of the patient, our institution uses a particular surgical protocol that has proven to be very effective to treat MAPE without recurring to deep hypothermia or circulatory arrest. To illustrate this protocol, the surgery of the above patient is described below.

Anesthesiologic preparation was made using the standardized protocols for cardiac surgeries. Continuous cerebral oximetry monitoring was used in order to assess brain perfusion during the CPB but, as deep hypothermia and circulatory arrest were not employed, cerebrospinal fluid pressure monitoring was not needed. This can further avoid risk of injury to the spinal cord.

Median sternotomy was performed, and the pericardium was opened wide using the inverted-T technique. To establish CPB, perfusion cannulae were placed into the upper ascending aorta in order to easily mobilize it and both caval veins. A left atrium vent was placed in order to decompress the left ventricle and to realize a "back aspiration" during the main procedure. After total CPB was achieved, the lungs were ventilated with low tidal volumes in order to reduce postoperative lung atelectasis until cooling at $24^{\circ} \mathrm{C}$ (rectal temperature) was completed. CPB was decreased until a perfusion flow of $1-1.2 \mathrm{~L} / \mathrm{min}$ was reached. This approach, together with mild hypothermia, allows the reduction of pulmonary backflow at the opening of the pulmonary arteries and the simultaneous maintenance of effective organ perfusion and a good surgical vision. Meanwhile, we mobilized the superior vena cava circumferentially, avoiding splitting the azygos or doing damage to the right phrenic nerve; in this way, the right pulmonary artery remains visible and, through the opening of right pleural space, it can be isolated up until the lobar and segmental branches. After this, modified Bretschneider cardioplegia was administered in antegrade fashion according to the manufacture's protocol. The right pulmonary artery was reached by moving the upper caval vein laterally and the aorta medially using a rubber covered retractor, with care taken not to damage the posterior wall of the aorta. The pulmonary artery was incised distally until the trifurcation and until $1 \mathrm{~cm}$ before the pulmonary trunk proximally (Figure 2). At this moment, if the thrombus is not fresh, it is critical to find the correct endarterectomy plane because a deeper plane risks damage to the vessel wall and causes difficulties in bleeding control. Usually, after reaching the pulmonary branches, the thrombus begins to be covered by platelets and fibrin that make it difficult to dissect it from the vessel wall (Figure 3). According to our protocol, the thrombus core was isolated circumferentially using a particular long-shaped dissector and removal was accomplished by extending the dissector first to the upper lobe and then to the other lobes. If blood occludes the operative field view, the surgical table can be to inclined laterally, towards one side or another. After the removal of the thrombus was complete, the incision was closed with a 6-0 polypropylene running suture (alternatively, an autologous pericardial patch could be used). The left pulmonary artery was reached by retracting the heart up and medially, as is done in coronary bypass surgery to reach 


\section{Page 4 of 7}

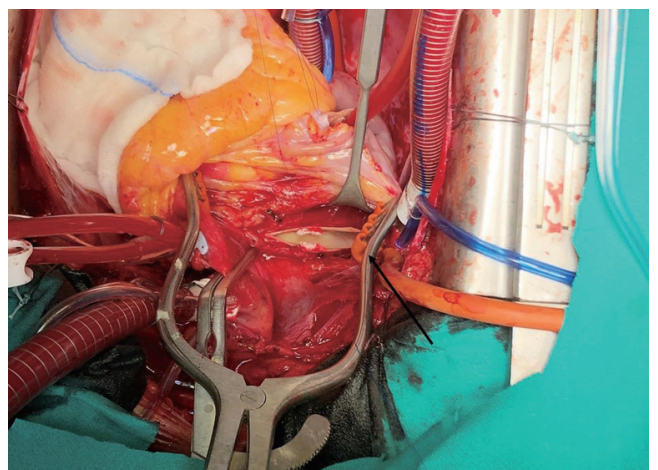

Figure 2 Longitudinal incision of the anterior wall of the left pulmonary artery. Margins are opened using 2 single 6-0 polypropylene stiches on PTFE pledgets. The artery was reached by moving the upper caval vein laterally and the aorta medially using a rubber covered retractor (arrow). PTFE, polytetrafluoroethylene.

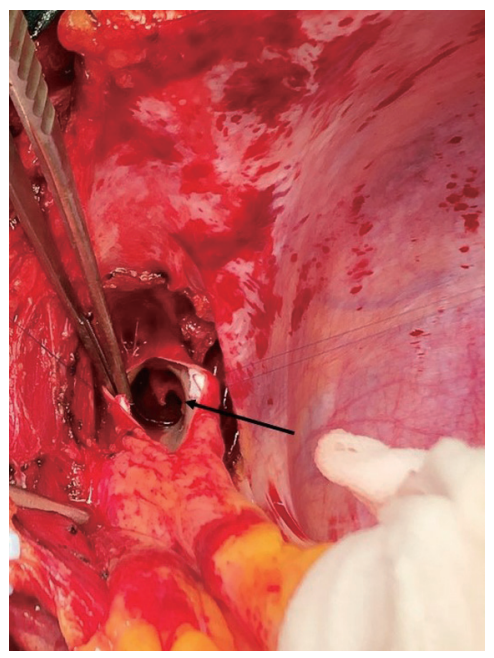

Figure 3 Intravascular vision of the thrombus partially detached from the arterial wall (arrow).

the lateral wall vessels (Figure 4). This technique is the same as that performed for the right pulmonary artery beginning from the upper and lingular branches. When thrombus removal was complete (Figure 5), a deairing procedure was performed and rewarming was started. As recommended by protocol, the patient was slowly weaned from CPB in order to clear out all the effects of prolonged hypothermia and ischemia. The serum lactate levels remained satisfactory during the CPB (CPB parameters are shown in Table 1) as did that of continuous cerebral saturation. Perfusion arterial pressure was always maintained around $45 \mathrm{mmHg}$ during the aortic cross clamping in order to balance organ

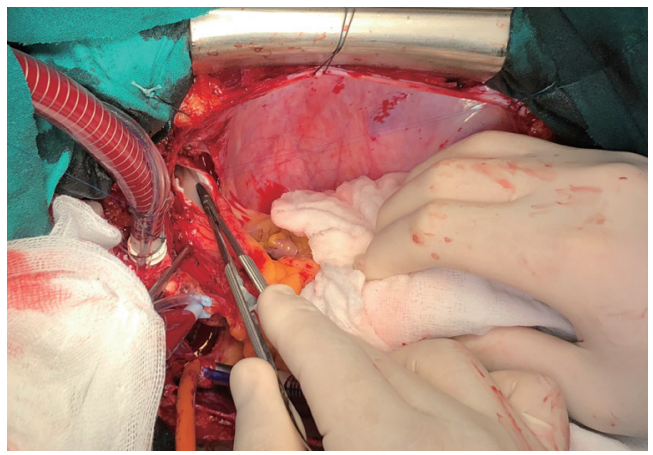

Figure 4 Heart displaced up and medially in order to expose the left pulmonary artery.

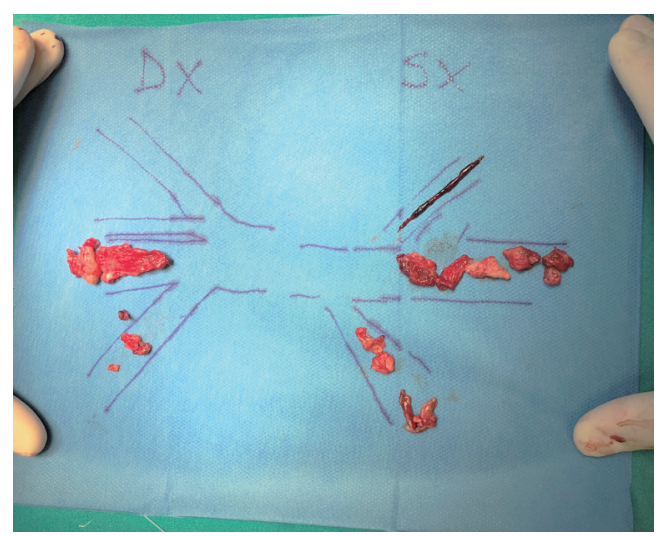

Figure 5 Complete pattern of the removed thrombus at the end of the procedure.

perfusion and risk of cerebral edema; in order to increase cerebral perfusion, arterial carbon dioxide pressure $\left(\mathrm{PCO}_{2}\right)$ was maintained at $40-45 \mathrm{mmHg}$. The total $\mathrm{CPB}$ time was 235 minutes, which included 143 minutes of total CPB and 120 minutes of aortic cross clamping. Using only mild hypothermia also avoids extreme hemodilution; in this case, the patient's hemoglobin was above $11 \mathrm{~g} / \mathrm{dL}$ during the duration of the CPB. After CPB weaning, the treatment course was uneventful.

\section{Postoperative management}

Postoperative management of MAPE patients is usually difficult, especially due to the length of surgery and CPB times. In this regard, avoiding hypothermic arrest allows for an accelerated postoperative recovery which reduces the negative effect of hypothermia on renal and cerebral function. In the postoperative period, our protocol 


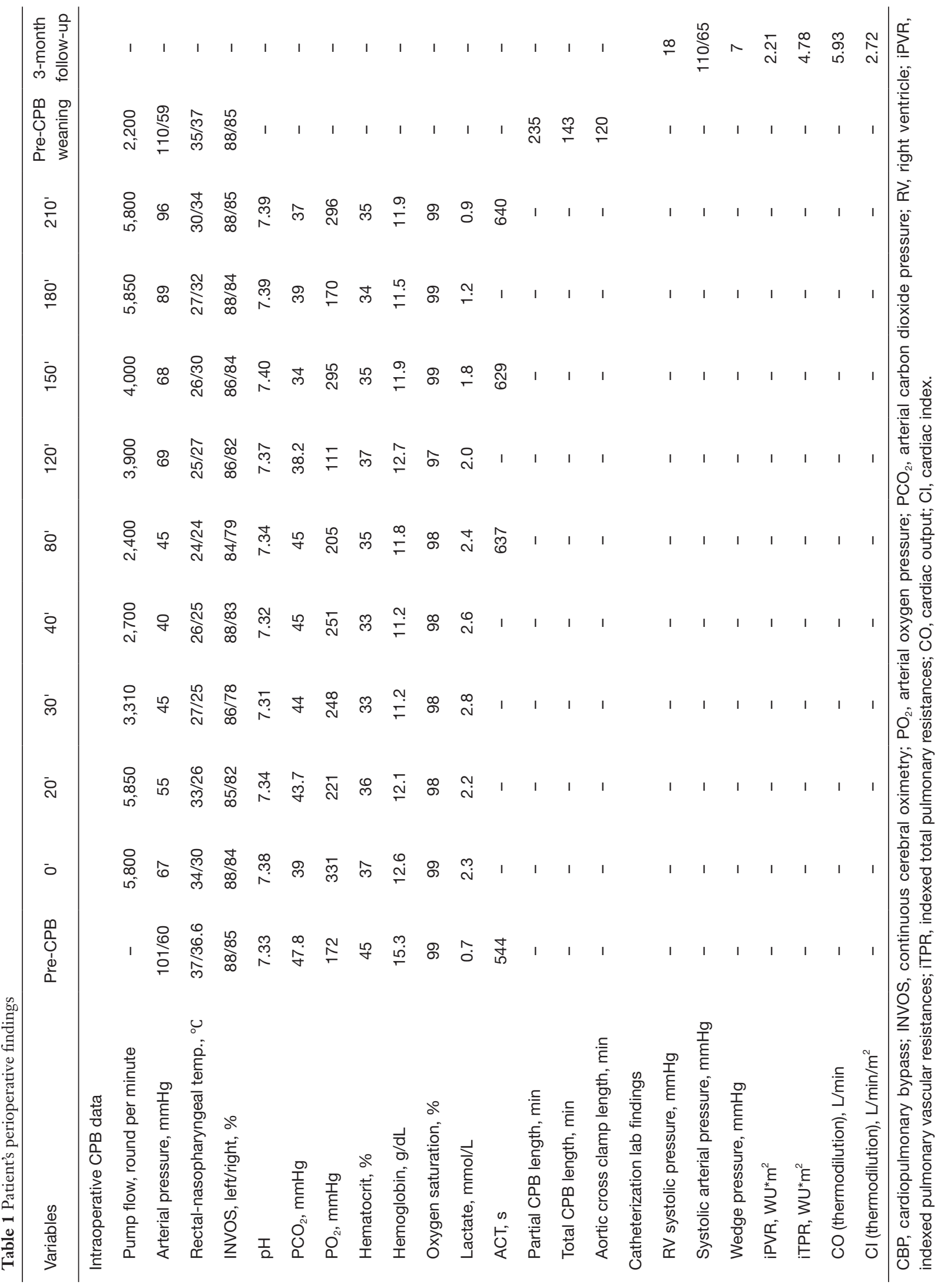


recommends slowly waking the patient and gradually weaning him or her from mechanical ventilation in order to allow a decrease of postreperfusion-related pulmonary edema and an increase in the patient's response. Infusion of prostaglandin $\mathrm{E}_{1}$ or inhaled nitric oxide (12) may be useful after the surgery especially when reactive postoperative pulmonary arterial hypertension occurs. If this does occur, a right heart angiographic study could be performed after recovery or at follow-up to assess pulmonary pressure and vascular resistance (the patient's results are shown in Table 1).

\section{Ethics and consent}

All procedures performed in this study were in accordance with the ethical standards of the institutional and/or national research committee(s) and with the Helsinki Declaration (as revised in 2013). Written informed consent was obtained from the patient for publication of this study and accompanying images. A copy of the written consent is available for review by the editorial office of this journal.

\section{Conclusions and comment}

Surgical treatment of MAPEs, without using deep hypothermia and circulatory arrest unless strictly necessary, has proven to be a very effective technique (13). Circulatory arrest requires very low body temperatures $\left(18-20^{\circ} \mathrm{C}\right)$ and long cooling-rewarming times that significantly increase the surgical length and the risks of renal and neurological injuries; in addition, circulatory arrest cannot be maintained for more than 30 minutes without damaging tissues, and so the surgeon is forced to rewarm the patient, reperfuse the patients for a period, and then recool them. This approach requires a considerable expenditure of time that can be avoided with the above-described technique. Indeed, our protocol reduces both the operation time and the subsequent postoperative recovery period in the ICU. In short, abbreviated CPB times lead to lower hemodilution and decreased inflammatory response, which in turn reduce the risk of cerebral, lung, and renal edema, and hasten ICU discharge.

\section{Acknowledgments}

Funding: None.

\section{Footnote}

Provenance and Peer Review: This article was commissioned by the guest editors (Andrea Dell'Amore and Nizar Asadi) for the series "Mechanical Extracorporeal CardioRespiratory Supports in General Thoracic Surgery" published in Current Challenges in Thoracic Surgery. The article has undergone external peer review.

Conflicts of Interest: Both authors have completed the ICMJE uniform disclosure form (available at https://ccts. amegroups.com/article/view/10.21037/ccts-20-84/coif). The series "Mechanical Extracorporeal Cardio-Respiratory Supports in General Thoracic Surgery" was commissioned by the editorial office without any funding or sponsorship. The authors have no other conflicts of interest to declare.

Ethical Statement: The authors are accountable for all aspects of the work in ensuring that questions related to the accuracy or integrity of any part of the work are appropriately investigated and resolved. All procedures performed in this study were in accordance with the ethical standards of the institutional and/or national research committee(s) and with the Helsinki Declaration (as revised in 2013). Written informed consent was obtained from the patient for publication of this study and accompanying images. A copy of the written consent is available for review by the editorial office of this journal.

Open Access Statement: This is an Open Access article distributed in accordance with the Creative Commons Attribution-NonCommercial-NoDerivs 4.0 International License (CC BY-NC-ND 4.0), which permits the noncommercial replication and distribution of the article with the strict proviso that no changes or edits are made and the original work is properly cited (including links to both the formal publication through the relevant DOI and the license). See: https://creativecommons.org/licenses/by-nc-nd/4.0/.

\section{References}

1. Goldhaber SZ, Visani L, De Rosa M. Acute pulmonary embolism: clinical outcomes in the International Cooperative Pulmonary Embolism Registry (ICOPER). Lancet 1999;353:1386-9.

2. Samoukovic G, Malas T, deVarennes B. The role of pulmonary embolectomy in the treatment of acute pulmonary embolism: a literature review from 1968 to 2008. Interact Cardiovasc Thorac Surg 2010;11:265-70.

3. Kalra R, Bajaj NS, Arora P, et al. Surgical Embolectomy for Acute Pulmonary Embolism: Systematic Review 
and Comprehensive Meta-Analyses. Ann Thorac Surg 2017;103:982-90.

4. Iaccarino A, Frati G, Schirone L, et al. Surgical embolectomy for acute massive pulmonary embolism: state of the art. J Thorac Dis 2018;10:5154-61.

5. Sista AK, Kuo WT, Schiebler M, et al. Stratification, Imaging, and Management of Acute Massive and Submassive Pulmonary Embolism. Radiology 2017;284:5-24.

6. Stein PD, Hull RD, Ghali WA, et al. Tracking the uptake of evidence: two decades of hospital practice trends for diagnosing deep vein thrombosis and pulmonary embolism. Arch Intern Med 2003;163:1213-9.

7. Aymard T, Kadner A, Widmer A, et al. Massive pulmonary embolism: surgical embolectomy versus thrombolytic therapy--should surgical indications be revisited?. Eur J Cardiothorac Surg 2013;43:90-4; discussion 94.

8. Cooley DA, Beall AC Jr, Alexander JK. Acute massive pulmonary embolism. Successful surgical treatment using temporary cardiopulmonary bypass. JAMA 1961;177:283-6.

doi: $10.21037 /$ ccts-20-84

Cite this article as: Pilato E, Comentale G. Surgical treatment of acute pulmonary embolism: a modified surgical technique to avoid deep hypothermia and circulatory arrest. Curr Chall Thorac Surg 2020;2:37.
9. Konstantinides SV, Meyer G, Becattini C, et al. 2019 ESC Guidelines for the diagnosis and management of acute pulmonary embolism developed in collaboration with the European Respiratory Society (ERS). Eur Heart J 2020;41:543-603.

10. Meneveau N, Séronde MF, Blonde MC, et al. Management of unsuccessful thrombolysis in acute massive pulmonary embolism. Chest 2006;129:1043-50.

11. Faluk M, Hasan SM, Chacko JJ, et al. Evolution of Acute Pulmonary Embolism Management: Review Article. Curr Probl Cardiol 2021;46:100551.

12. Kline JA, Hall CL, Jones AE, et al. Randomized trial of inhaled nitric oxide to treat acute pulmonary embolism: The iNOPE trial. Am Heart J 2017;186:100-10.

13. Van Putte BP, Bantal N, Snijder R, et al. Acute massive pulmonary embolism treated by thrombo-embolectomy using intermittent deep hypothermic circulatory arrest. Interact Cardiovasc Thorac Surg 2008;7:412-3.

(English Language Editor: J. Gray) 